\title{
A Penrose-like inequality for maximal asymptotically flat spin initial data sets
}

\author{
Daniel Maerten
}

Received: 3 October 2006 / Accepted: 2 March 2007 / Published online: 26 April 2007

(C) Springer Science+Business Media B.V. 2007

\begin{abstract}
We prove a Penrose-like inequality for the mass of a large class of constant mean curvature (CMC) asymptotically flat $n$-dimensional spin manifolds which satisfy the dominant energy condition and have a future converging, or past converging compact and connected boundary of non-positive mean curvature and of positive Yamabe invariant. We prove that for every $n \geq 3$ the mass is bounded from below by an expression involving the norm of the linear momentum, the volume of the boundary, dimensionless geometric constants and some normalized Sobolev ratio.
\end{abstract}

Keywords Penrose inequality - Asymptotically flat manifolds · Mass · Linear momentum $\cdot$ Conformal methods $\cdot$ Dirac operator $\cdot$ Spinors

\section{Introduction}

The energy-momentum is a global Riemannian invariant of asymptotically flat manifolds which was defined by physicists in the General Relativity context [33]. The positive mass conjecture is one of the most important issues involving the energymomentum and has been proved by Schoen and Yau [29-31] (for dimension $3 \leq n \leq 7$ ) with minimal surface arguments, and also by Witten [35] (cf. also Parker and Taubes' proof [27]) in any dimension on spin manifolds.

In 1973, Penrose [28] stated a refinement of the positive mass conjecture for Riemannian asymptotically flat 3-manifolds (without extrinsic curvature).

Riemannian Penrose inequality Let $(M, g)$ be a 3-dimensional asymptotically flat Riemannian manifold with a compact, connected, outermost minimal boundary $\partial M$ (which is a topological 2-sphere). Suppose also that the scalar curvature of $(M, g)$ is non-negative. Then its mass $m$, if defined, satisfies

\footnotetext{
D. Maerten $(\bowtie)$

Institut de Mathématiques, Université de Neuchâtel,

Rue Emile Argand 11, CP 158,

2009 Neuchatel, Switzerland

e-mail: daniel.maerten@unine.ch
} 


$$
m \geq \frac{1}{4} \sqrt{\frac{\operatorname{Area}(\partial M, g)}{\pi}},
$$

and equality is achieved if and only if $(M, g)$ is a spacelike Schwarzschild metric.

The Riemannian Penrose Inequality [10] has been proved, in dimension 3, by Huisken and Ilmanen [20,21] using the inverse mean curvature flow, and independently by Bray [9] using the positive mass theorem. In [16], Herzlich proved, still in dimension 3, an optimal Penrose-like inequality (with a constant) involving the area of the boundary $\partial M$ and some normalized Sobolev ratio. He recently improved this result [17] in higher dimensions for asymptotically flat spin manifolds that have a compact and connected boundary of positive Yamabe invariant, which is mainly the case for outer apparent horizons, according to a new result of Galloway and Schoen [14] (their result generalizes Hawking's one to higher dimensions).

Now, a natural idea is to extend the statement of the Riemannian Penrose Inequality in the context of asymptotically flat spin $n$-manifolds $(M, g, k)(n \geq 3)$ endowed with a non-zero extrinsic curvature tensor $k$ [24]. One of the difficulties of this issue is to formulate a plausible conjecture. The fact that the extrinsic curvature $k$ is nonzero, and considering higher dimensions make the context of the Penrose inequality almost unknown at the time of writing of this work. However, this question seems to be of physical great interest according to the existence of a counterexample of the Riemannian Penrose inequality with charge [34] in dimension 3.

The aim of the present paper is to prove a Penrose-like inequality for a large class of CMC asymptotically flat, $n$-dimensional and spin manifolds with boundary $(M, g, k)$, which generalizes Herzlich's one [16,17]. More precisely, $(M, g, k)$ is asymptotically flat if there exist some compact $K$, a positive number $R$ and a diffeomorphism called a chart at infinity $M \backslash K \longrightarrow \mathbb{R}^{n} \backslash B(0, R)$ such that in this chart we have

$$
e:=g-b \in C_{\beta}^{2, \alpha}, \quad k \in C_{-1+\beta}^{1, \alpha},
$$

where $b$ denotes the Euclidean background metric and $C_{\beta}^{k, \alpha}$ are the weighted Hölder spaces (of functions, tensors or spinors according to the context) defined by

$$
C_{\beta}^{k, \alpha}=\left\{w \in C_{\mathrm{loc}}^{k, \alpha},\left\|(1+r)^{-\beta+\alpha+k}\left[D^{k} w\right]_{\alpha}\right\|_{C^{0}}+\sum_{p=0}^{k}\left\|(1+r)^{-\beta+p} D^{p} w\right\|_{C^{0}}<\infty\right\} .
$$

In this definition, $D$ denotes the flat Levi-Civita connection and

$$
\left[D^{k} w\right]_{\alpha}:=\sup _{\left|z-z^{\prime}\right| \leq 1}\left\{\left|z-z^{\prime}\right|^{-\alpha}\left|D^{k} w(z)-D^{k} w\left(z^{\prime}\right)\right|\right\}
$$

for $z=\left(z^{i}\right)$ any system of coordinates at infinity $(r=|z|)$. In this situation, $(M, g, k)$ will be said to be $C_{\beta}^{k, \alpha}$-asymptotically flat. Analogously, $(M, g, k)$ is $W_{\beta}^{s, p}$-asymptotically flat, $s \in \mathbb{N}, p \geq 2$, if

$$
e:=g-b \in W_{\beta}^{s, p}, \quad k \in W_{-1+\beta}^{s, p},
$$

where $W_{\beta}^{s, p}$ are the Sobolev spaces (of functions, tensors or spinors according to the context) defined by

$$
W_{\beta}^{s, p}=\left\{w \in W_{\text {loc }}^{s, p},(1+r)^{-\beta-\frac{n}{p}+j} D^{j} w \in L^{p} \quad \forall j \leq s\right\} .
$$


The weighted indexing convention is convenient for the Sobolev spaces since the value of $\beta$ gives the asymptotic behaviour at infinity. We will refer to [2] for basic properties of these spaces. In the remainder of the paper we will use the usual equivalent norm on the Sobolev function space $W_{-\frac{(n-2)}{2}}^{1,2}$ given by $\int_{M}|\mathrm{~d} w|^{2}$.

Let us assume furthermore that $\beta<-\frac{(n-2)}{2}$ and the constraints

$$
\Phi(g, k):=\left(\begin{array}{c}
\mathrm{Scal}^{g}+\left(\operatorname{tr}_{g} k\right)^{2}-|k|_{g}^{2} \\
2\left(\delta_{g} k+\operatorname{dtr}_{g} k\right)
\end{array}\right) \in L^{1}\left(M, \mathrm{dVol}_{g}\right),
$$

where $\delta_{g}$ is the $g$-divergence (with a - sign in front of it). Then $p^{\mu} \in \mathbb{R}^{n, 1}$ the energymomentum of $(g, k)$ is (well-) defined [2] as

$$
\begin{aligned}
m=p^{0} & =\frac{1}{16 \pi} \lim _{r \rightarrow \infty} \int_{S_{r}(b)}(\delta g+\mathrm{dtr} g)\left(v_{r}\right) \mathrm{dVol}_{S_{r}(b)}, \\
p^{i} & =\frac{1}{8 \pi} \lim _{r \rightarrow \infty} \int_{S_{r}(b)}\left(k\left(v_{r}, e_{i}\right)-g\left(v_{r}, e_{i}\right) \operatorname{tr} k\right) \mathrm{dVol}_{S_{r}(b)},
\end{aligned}
$$

where $\delta$ is the $b$-divergence (with a - sign in front of it), traces are taken with respect to $b, S_{r}(b)$ is a $b$-geodesic sphere of radius $r$ and $v_{r}$ its pointing toward infinity unit normal. The positive energy theorem proved in [18] (the proof is in dimension $n=3$ but it can be extended with no difficulty to higher dimensions under the condition that the manifold is spin) asserts that if $\left(M^{n}, g, k\right)$ satisfies the dominant energy condition

$$
\mathrm{Scal}^{g}+\left(\operatorname{tr}_{g} k\right)^{2}-|k|_{g}^{2} \geq 2\left|\delta_{g} k+\operatorname{dtr}_{g} k\right|_{g},
$$

and if $\partial M$ is composed with a finite number of either past or future trapped closed $(n-1)$-manifolds then $p^{\mu}$ is causal positively oriented. If we are in the border line case where $p^{\mu}$ is null then (cf. $\left.[7,13]\right)(M, g, k)$ is isometrically embeddable in Minkowski space-time.

We introduce additional definitions and notations for later use. Let $\left(N^{n+1}, \gamma\right)$ be a Lorentzian manifold of signature $(-,+, \ldots,+) .\left(M^{n}, g, k\right)$ is assumed to be a spin (in dimension 3 this only means orientable) Riemannian hypersurface whose induced metric is $g$ and second fundamental form (extrinsic curvature) is $k$. We will work with the complex spinor [22] bundle of $N$ restricted to the hypersurface $M$, that is to say $\Sigma:=\Sigma(N)_{\mid M}$ which is given by the choice of a unit normal $e_{0}$ of $M$ in $N$. More precisely, if one denotes by $P_{\operatorname{Spin}(n, 1)}(N)$ the bundle of $\operatorname{Spin}(n, 1)$-frames on $N$, and by $\rho_{n, 1}$ the standard representation of $\operatorname{Spin}(n, 1)$ then

$$
\Sigma(N):=P_{\operatorname{Spin}(n, 1)}(N) \times_{\rho_{n, 1}} \mathbb{C}^{[(n+1) / 2]} .
$$

Now the choice of unit normal $e_{0}$ of $M$ in $N$ induces a natural inclusion of Lie groups $\operatorname{Spin}(n) \subset \operatorname{Spin}(n, 1)$ and so we can define

$$
\Sigma:=P_{\operatorname{Spin}(n, 1)}(N) \times{ }_{\left(\rho_{n, 1}\right)_{\mid \operatorname{Spin}(n)}} \mathbb{C}^{[(n+1) / 2]} .
$$

$\Sigma$ naturally carries two sesquilinear inner products: the first one denoted by $(*, *)$ which is $\operatorname{Spin}(n, 1)$-invariant (it is not necessary positive); the second one denoted by $\langle *, *\rangle:=\left(e_{0} \cdot *, *\right)$ which is $\operatorname{Spin}(n)$-invariant and Hermitian definite positive $(\cdot$ is the Clifford action with respect to the metric $\gamma)$. The Hermitian or anti-Hermitian character of the Clifford multiplication by vectors differs if we consider $(*, *)$ or $\langle *, *\rangle$ 
and is described in [23], for instance. $\Sigma$ is also endowed with two different connections $\nabla^{\gamma}, \nabla^{g}$ which are respectively the Levi-Civita connections of $\gamma$ and $g$. Let us take a spinor field $\psi \in \Gamma(\Sigma)$ and a vector field $X \in \Gamma(T M)$, then our conventions are

$$
\begin{aligned}
\nabla_{X}^{\gamma} \psi & =\nabla_{X}^{g} \psi-\frac{1}{2} k(X) \cdot e_{0} \cdot \psi, \\
\langle k(X), Y\rangle_{\gamma} & =\left\langle\nabla_{X}^{\gamma} Y, e_{0}\right\rangle_{\gamma} .
\end{aligned}
$$

In these formulae denotes the Clifford action with respect to the metric $\gamma$. We will suppose that $M$ has a compact and connected boundary $\partial M$ whose induced metric, Levi-Civita connection and second fundamental form are respectively denoted by $\ell, \nabla^{\ell}, \theta$. Our conventions are

$$
\begin{aligned}
& \nabla_{X}^{g} Y=\nabla_{X}^{\ell} Y-\theta(X, Y) v, \\
& \nabla_{X}^{g} \psi=\nabla_{X}^{\ell} \psi-\frac{1}{2} \theta(X) \cdot v \cdot \psi,
\end{aligned}
$$

where $v$ is the unit normal to $\partial M$ pointing toward infinity (that is to say pointing inside $M)$, and $\cdot$ still denotes the Clifford action with respect to the metric $\gamma$.

We define the Yamabe invariant of the boundary $\partial M$ by

$$
\mathscr{Y}=\mathscr{Y}(\partial M, \ell):=\inf _{\bar{\ell} \in[\ell]}\left\{\frac{\int_{\partial M} \mathrm{Scal}^{\bar{\ell}} \mathrm{dVol}_{\bar{\ell}}}{\operatorname{Vol}(\partial M, \bar{\ell})^{\frac{n-3}{n-1}}}\right\},
$$

where $[\ell]$ is the conformal class of the boundary metric $\ell$. It will also be convenient to set $C_{n}:=4\left(\frac{n-1}{n-2}\right)$. The first result of this paper is a positive energy theorem [15] for asymptotically flat (in the Sobolev classes) spin $n$-manifolds with compact, connected boundary (which is an analogous version of Theorem 11.4 of [4]) using a spectral boundary condition. Note that the following result is true even for non-CMC manifolds.

Theorem 1.1 (Positive Energy) Let $\left(M^{n}, g, k\right)$ be a complete, spin, $W_{\beta}^{2,2}$-asymptotically flat manifold, $\beta<-\frac{(n-2)}{2}, n \geq 3$, with $\mathscr{Y}(\partial M, \ell)>0$, satisfying the dominant energy condition and the boundary condition

$$
\operatorname{tr}_{\ell} \theta+\left(\left(k(v, v)-\operatorname{tr}_{g} k\right)^{2}+\sum_{j=2}^{n} k\left(v, e_{j}\right)^{2}\right)^{1 / 2} \leq \frac{1}{2} \operatorname{Vol}(\partial M, \ell)^{\frac{-1}{n-1}} \sqrt{C_{n} \mathscr{Y}(\partial M, \ell)}
$$

Assume furthermore that the constraints $\Phi(g, k)$ are $L^{1}\left(M, \mathrm{dVol}_{g}\right)$.

Then the energy-momentum $p^{\mu}$ of $(g, k)$ is causal and future oriented.

In the remainder of this introduction, we will make the $\mathrm{CMC}$ assumption $\left(\operatorname{tr}_{g} k\right.$ is a constant), which in asymptotically flat geometry reduces to $\operatorname{tr}_{g} k=0$. Since the manifold $(M, g, k)$ is thought as an isometrically embedded space-like hypersurface in a Lorentzian manifold $(N, \gamma)$, then the CMC assumption gives the maximality of $(M, g, k)$ as Riemannian hypersurface of this Lorentzian manifold (see [3,5] for existence results of maximal hypersurfaces in asymptotically flat space-times). 
The compact and connected boundary $\partial M$ is supposed to be a weakly future trapped (respectively weakly past trapped) $(n-1)$-manifold which means that its mean curvature vector is causal and past (resp. future) pointing (cf. [26] for a precise definition). In our conventions, it can be expressed by the relation

$$
\operatorname{tr}_{g} k-k(v, v)=-k(v, v) \geq\left|\operatorname{tr}_{\ell} \theta\right| \quad\left(\text { resp. }-\operatorname{tr}_{g} k+k(v, v)=k(v, v) \geq\left|\operatorname{tr}_{\ell} \theta\right|\right) .
$$

$\partial M$ is also assumed to have non-positive mean curvature $\operatorname{tr}_{\ell} \theta \leq 0$. This assumption is motivated by the fact that an apparent horizon has non-positive mean curvature since it satisfies the more restrictive conditions

$$
\operatorname{tr}_{g} k-k(v, v)=-k(v, v) \geq \operatorname{tr}_{\ell} \theta, \quad \operatorname{tr}_{g} k-k(v, v)=-k(v, v)=-\operatorname{tr}_{\ell} \theta .
$$

We also set $k(v)=\sum_{j=1}^{n} k\left(v, e_{j}\right) e_{j}$ for any local orthonormal frame $\left(e_{j}\right)_{j=1}^{n}$, and

$$
V:=\operatorname{Vol}(\partial M, \ell), \Theta:=V^{\frac{1}{n-1}} \sup _{\partial M}\left\{\operatorname{tr}_{\ell} \theta+|k(v)|_{g}\right\},
$$

The normalized Sobolev ratio is finally defined by

$$
\mathfrak{S}:=C_{n} V^{\frac{1}{n-1}} \inf _{\substack{1,2 \\ W_{-\frac{(n-2)}{2}}}}\left\{\frac{\int_{M}|\nabla f|^{2}}{\int_{\partial M} f^{2}}\right\}
$$

We can now state the main results of this paper.

Theorem 1.2 Let $(M, g, k)$ be a $n$-dimensional, complete, $C_{\tau}^{2, \alpha}$-asymptotically flat $\left(\tau<-\frac{n-2}{2}, n \geq 3\right), C M C$ and spin manifold with constraints satisfying the dominant energy condition and $\Phi(g, k) \in L^{1}\left(M, \mathrm{dVol}_{g}\right)$. Suppose furthermore that $M$ has a compact, connected and weakly future (resp. past) trapped boundary $\partial M$ of positive Yamabe invariant and of non-positive mean curvature satisfying

$$
\Theta<\frac{1}{2} \sqrt{C_{n} \mathscr{Y}} .
$$

Then there exists (an explicit definition will be given in Sect. 4) a small positive constant $\zeta_{0}=\zeta_{0}(M, g)$, such that $p^{\mu}=\left(m, p^{i}\right)$ the energy-momentum of $(g, k)$ satisfies

$$
m \geq \zeta_{0} \sqrt{\sum_{i=1}^{n}\left(p^{i}\right)^{2}}+\frac{\mathfrak{S}\left(\sqrt{\mathscr{Y} C_{n}}-2 \Theta\right)}{\mathfrak{S}+\left(\sqrt{\mathscr{Y} C_{n}}-2 \Theta\right)}\left(\frac{V^{\frac{4}{C_{n}}}}{16 \pi}\right)
$$

\section{Remark 1.3}

1. It would be nice to prove (1) with $\zeta_{0}=1$ in order to obtain a Lorentzianlooking inequality that would clearly be a refinement of the positive energy theorem for manifolds with boundary and so be a relevant conjecture for the Penrose inequality in case of $k \neq 0$.

2. The dominant energy condition and the $L^{1}$ character of the constraints can be replaced by the more restrictive condition: $(M, g, k)$ is an initial data set, that is to say $\Phi(g, k)=0$.

3. $(M, g, k)$ clearly satisfies the assumptions of Theorem 1.1 . 
Under the CMC assumption, the constant $\Theta$ of our theorem is non-negative since the fact that the boundary $\partial M$ is weakly future (resp. past) trapped ensures the nonnegativity of the function $\operatorname{tr}_{g} \theta+|k(v)|_{g}$ along $\partial M$. It is also important to notice the scale-invariance of the constant $\Theta$ (this is also the case of the constant $\zeta_{0}(M, g)$ which will be defined in Sect. 4). More precisely, if one considers the harmless rescaling of initial data sets which are given by the conformal transformation $\Lambda_{t}:(g, k) \mapsto\left(t^{2} g, t k\right)$, for any constant and positive real parameter $t$, then $\Theta$ is invariant under each $\Lambda_{t}$. Surprisingly, one can find some triple $(M, g, k)$ with $k \neq 0$, satisfying the assumptions of Theorem 1.2 and $\Theta=0$ (It is sufficient to construct suitable traceless transverse tensor $k$ with the good boundary conditions. See [25] for a precise discussion of this point). This is the reason why Theorem 1.2 can be considered as a refinement of the Penroselike inequality of $[16,17]$ for a class of non-zero extrinsic curvature asymptotically flat manifolds.

Theorem 1.4 Suppose that the following conditions are satisfied:

1. The assumptions of Theorem 1.2 hold,

2. $g$ and $k$ are smooth,

3. Additional technical assumption: the symmetric 2-tensor $k$ restricted to TวM vanishes, namely $k^{\partial M}=0$.

If equality is achieved in (1), then $k=0$ on the whole manifold $M$, and $(M, g)$ is isometric to the standard spacelike $n$-slice in the exterior Schwarzschild metric of mass $m$, $M=\mathbb{R}^{n} \backslash B(0, R)$ with

$$
R=\left(\frac{4 \pi m}{(n-1) \omega_{n-1}}\right)^{\frac{1}{n-2}} \text { and } g=\left(1+\left(\frac{R}{r}\right)^{n-2}\right)^{\frac{4}{n-2}} \text { eucl. }
$$

It is quite satisfactory that we recover the same conclusion as in $[16,17]$ when equality is achieved, even if we have to make assumption 3 . This means that our Penrose-like inequality is somehow optimal in the non-empty (this point will be shown in the appendix) class of CMC asymptotically flat spin manifolds under consideration.

In dimension 3, the Yamabe number of the closed surface $\partial M$ reduces to a topological invariant because of the Gauss-Bonnet formula which relates the total scalar curvature to the Euler class. More precisely, Theorem 1.2 becomes in dimension 3.

Theorem 1.5 Let $(M, g, k)$ be a 3-dimensional, complete, $C_{\tau}^{2, \alpha}$-asymptotically flat $\left(\tau<-\frac{1}{2}\right), C M C$ and orientable manifold with constraints satisfying the dominant energy condition and $\Phi(g, k) \in L^{1}\left(M, \mathrm{dVol}_{g}\right)$. Suppose furthermore that $M$ has a compact, connected and weakly future (resp. past) trapped boundary $\partial M$ of non-positive mean curvature, which satisfies in addition $\Theta<4 \sqrt{\pi}$.

Then there exists a small positive constant $\zeta_{0}=\zeta_{0}(M, g)$, such that $p^{\mu}=\left(m, p^{i}\right)$ the energy-momentum of $(g, k)$ satisfies

$$
m \geq \zeta_{0} \sqrt{\sum_{i=1}^{3}\left(p^{i}\right)^{2}}+\frac{\mathfrak{S}(8 \sqrt{\pi}-2 \Theta)}{\mathfrak{S}+(8 \sqrt{\pi}-2 \Theta)}\left(\frac{\sqrt{A}}{16 \pi}\right) .
$$

In the context of the generalized Penrose inequality, it seems to be of interest to insist on the following straightforward consequence. 
Corollary 1.6 For any manifolds $\left(M^{n}, g, k\right)$ satisfying the assumptions of Theorem 1.2, the mass, if defined, satisfies

$$
m \geq \frac{\mathfrak{S}\left(\sqrt{\mathscr{Y} C_{n}}-2 \Theta\right)}{\mathfrak{S}+\left(\sqrt{\mathscr{Y} C_{n}}-2 \Theta\right)}\left(\frac{V^{\frac{4}{C_{n}}}}{16 \pi}\right)
$$

This paper is organized as follows: In Sect. 2, we give the proof of the positive energy Theorem 1.1 for (not necessary $C M C$ ) asymptotically flat spin manifolds with boundary which mainly relies on recent results of [4]. In Sect. 3 we make a conformal change of metric and second fundamental form à la Choquet Bruhat-LichnerowiczYork $[6,12,25]$ in order to solve some differential equations and obtain the conditions of application of the positivity result of the previous section. We give the last steps of the proof of Theorems 1.2 and 1.4 in Sect. 4. The last section is an appendix where we show, by constructing an example, that the class of asymptotically flat manifolds under consideration in this paper is non-empty.

\section{Positive energy theorem for manifolds with boundary}

The aim of this section is to prove the positive energy Theorem 1.1 for asymptotically flat manifolds (in the Sobolev classes) with compact connected boundary (which is an analogous version of Theorem 11.4 of [4]) using a spectral boundary condition. Note that the following result holds even for non-CMC manifolds.

Theorem 1.1 (Positive Energy) Let $\left(M^{n}, g, k\right)$ be a complete, spin, $W_{\beta}^{2,2}$-asymptotically flat manifold, $\beta<-\frac{(n-2)}{2}$, with $\mathscr{Y}(\partial M, \ell)>0$, satisfying the dominant energy condition and the boundary condition

$$
\operatorname{tr}_{\ell} \theta+\left(\left(k(v, v)-\operatorname{tr}_{g} k\right)^{2}+\sum_{j=2}^{n} k\left(v, e_{j}\right)^{2}\right)^{1 / 2} \leq \frac{1}{2} \operatorname{Vol}(\partial M, \ell)^{\frac{-1}{n-1}} \sqrt{C_{n} \mathscr{Y}(\partial M, \ell)} .
$$

Assume furthermore that the constraints $\Phi(g, k)$ are $L^{1}\left(M, \mathrm{dVol}_{g}\right)$.

Then the energy-momentum $p^{\mu}$ of $(g, k)$ is causal and positively oriented.

Proof Let $\left(e_{k}\right)_{k=0}^{n}$ is an orthonormal basis at the point with respect to the metric $\gamma$. We define respectively the Dirac-Witten operator of $M$ and the Dirac operator of $\partial M$

$$
\mathfrak{D}^{\gamma} \psi=\sum_{k=1}^{n} e_{k} \cdot N \nabla_{e_{k}}^{\gamma} \psi, \quad \mathfrak{D}^{\ell} \psi=\sum_{k=2}^{n} e_{k} \cdot \partial M \nabla_{e_{k}}^{\ell} \psi .
$$

where $\cdot N=\cdot, \cdot M$ and $\cdot \partial M$ respectively denote the Clifford actions of respectively $\gamma, g$ and $\ell$. The Bochner-Lichnerowicz-Weitzenbock-Witten formula is

$$
\left(\mathfrak{D}^{\gamma}\right)^{*} \mathfrak{D}^{\gamma}=\left(\mathfrak{D}^{\gamma}\right)^{2}=\left(\nabla^{\gamma}\right)^{*} \nabla^{\gamma}+\mathfrak{R},
$$

where $\Re:=\frac{1}{4}\left(\operatorname{Scal}^{\gamma}+4 \mathrm{Ric}^{\gamma}\left(e_{0}, e_{0}\right)+2 e_{0} \cdot \operatorname{Ric}^{\gamma}\left(e_{0}\right)\right)$. As usual we derive an integration by parts formula

$$
\begin{aligned}
\int_{M_{r}}\left|\mathfrak{D}^{\gamma} \psi\right|^{2}= & \int_{M_{r}}\left(\left|\nabla^{\gamma} \psi\right|^{2}+\langle\mathfrak{R} \psi, \psi\rangle\right)+\int_{\partial M}\left\langle\nabla_{\nu}^{\gamma} \psi+v \cdot \mathfrak{D}^{\gamma} \psi, \psi\right\rangle \mathrm{dVol}_{\partial M} \\
& -\int_{S_{r}}\left\langle\nabla_{\nu_{r}}^{\gamma} \psi+v_{r} \cdot \mathfrak{D}^{\gamma} \psi, \psi\right\rangle \mathrm{dVol}_{S_{r}(g)} .
\end{aligned}
$$


Now expressing $\nabla^{\gamma}$ in term of the $(n-1)$-dimensional connection and second form, and the $n$-dimensional second form, one gets for any $\psi \in \Gamma\left(\Sigma_{\mid \partial M}\right)$

$\nabla_{\nu}^{\gamma} \psi+v \cdot \mathfrak{D}^{\gamma} \psi=-\mathfrak{D}^{\ell} \psi+\frac{1}{2} e_{0} \cdot\left\{-\left(\operatorname{tr}_{\ell} \theta\right) e_{0}+\left(k(v, v)-\operatorname{tr}_{g} k\right) v+\sum_{j=2}^{n} k\left(v, e_{j}\right) e_{j}\right\} \cdot \psi$,

where we have used the following isomorphisms $X \cdot \partial M \psi=X \cdot M v \cdot M \psi$, and $X \cdot M \psi=\mathbf{i} X \cdot{ }_{N} e_{0} \cdot N \psi$, so as to identify $\mathfrak{D}^{\ell}$. It is then natural to define the vector field $\mathbf{k} \in \Gamma\left(T N_{\mid M}\right)$ by

$$
\mathbf{k}:=-\left(\operatorname{tr}_{\ell} \theta\right) e_{0}+\left(k(v, v)-\operatorname{tr}_{g} k\right) v+\sum_{j=2}^{n} k\left(v, e_{j}\right) e_{j} .
$$

For any smooth and compactly supported spinor $\psi$ we have then

$$
\int_{M}\left|\mathfrak{D}^{\gamma} \psi\right|^{2}=\int_{M}\left(\left|\nabla^{\gamma} \psi\right|^{2}+\langle\mathfrak{R} \psi, \psi\rangle\right)+\int_{\partial M}\left\langle-\mathfrak{D}^{\ell} \psi+\frac{1}{2} e_{0} \cdot \mathbf{k} \cdot \psi, \psi\right\rangle .
$$

As in [16], we denote by $P_{ \pm}$the $L^{2}$-orthogonal projections on the spaces of eigenvectors of positive (respectively negative) eigenvalues of $\mathfrak{D}^{\ell}$ on $\partial M$. We also define the Hilbert space

$$
\mathscr{H}=\left\{\psi \in W_{-\frac{(n-2)}{2}}^{1,2}(\Sigma), P_{+} \psi=0\right\} .
$$

Let us take $\psi \in C_{c}^{\infty}$ (which is dense in $W_{-\frac{(n-2)}{2}}^{1,2}$ ) such that $P_{+} \psi=0$ and compute

$$
\int_{\partial M}\left\langle\nabla_{v}^{\gamma} \psi+v \cdot \mathfrak{D}^{\gamma} \psi, \psi\right\rangle \mathrm{dVol}_{\partial M}=\sum_{m<0} \int_{\partial M}\left\langle\left(-\lambda_{m}+\frac{1}{2} e_{0} \cdot \mathbf{k} \cdot\right) \psi_{m}, \psi_{m}\right\rangle,
$$

where $\left(\lambda_{m}, \psi_{m}\right)_{m \in \mathbb{Z}^{*}}$ are the eigenvalues and eigenspinors of $\mathfrak{D}^{\ell}$ (with the convention that $\lambda_{m}<0$ if and only if $\left.m<0\right)$. But $-2 \lambda_{m}+e_{0} \cdot \mathbf{k} \cdot$ is a spinorial endomorphism which is non-negative if and only if the vector field $\left(\mathbf{k}-2 \lambda_{m} e_{0}\right)$ is causal future i.e.

$$
-2 \lambda_{m} \geq \operatorname{tr}_{\ell} \theta+\left(\left(k(v, v)-\operatorname{tr}_{g} k\right)^{2}+\sum_{j=2}^{n} k\left(v, e_{j}\right)^{2}\right)^{1 / 2} .
$$

As a consequence, using the estimate of Bär and Hijazi [1,19] this boundary integral is non-negative as soon as

$$
\begin{aligned}
\operatorname{tr}_{\ell} \theta+\left(\left(k(v, v)-\operatorname{tr}_{g} k\right)^{2}+\sum_{j=2}^{n} k\left(v, e_{j}\right)^{2}\right)^{1 / 2} & \leq \frac{1}{2} \operatorname{Vol}(\partial M, g)^{\frac{-1}{n-1}} \sqrt{C_{n} \mathscr{Y}(\partial M, \ell)} \\
& \leq 2 \lambda:=2 \inf _{m<0}\left\{-\lambda_{m}\right\} .
\end{aligned}
$$

Now the sesquilinear form $\int_{M}\left\langle\mathfrak{D}^{\gamma} *, \mathfrak{D}^{\gamma} *\right\rangle$ is clearly coercive on $C_{c}^{\infty}$ (because of the dominant energy condition and the estimate above) and also on $\mathscr{H}\left(\equiv \mathbb{H}_{P_{+}}\right.$in the notation of [4]) according to Lemma 11.1 and Theorem 11.4 of [4]. Consider now a smooth spinor field $\psi_{0}$ which is constant around infinity (in some chart at infinity) 
and satisfies $P_{+} \psi_{0}=0$. Using the coercivity, we can find (cf. also Theorem 8.8 of [4]) a $\psi \in \mathscr{H}$ so that $\mathfrak{D}^{\gamma}\left(\psi+\psi_{0}\right)=0$, and $P_{+}\left(\psi+\psi_{0}\right)=0$. Thereby one obtains

$$
\left.4 \pi\left\langle\psi_{0}, e_{0} \cdot p \cdot \psi_{0}\right\rangle=\lim _{r \rightarrow \infty} \int_{S_{r}(b)}\left\langle\nabla_{v_{r}}^{\gamma}\left(\psi_{0}+\psi\right)+v_{r} \cdot \mathfrak{D}^{\gamma}\left(\psi_{0}+\psi\right), \psi_{0}+\psi\right)\right\rangle \geq 0,
$$

which implies that the energy-momentum $p^{\mu}$ is causal and positively oriented.

\section{Conformal changes}

In this section, we consider a complete, $\mathrm{CMC}, C_{\tau}^{2, \alpha}$-asymptotically flat spin manifold $\left(M^{n}, g, k\right)$, with $\tau<-\frac{(n-2)}{2}$. We assume that the dominant energy condition is satisfied and the constraints $\Phi(g, k) \in L^{1}\left(M, \mathrm{dVol}_{g}\right)$. The boundary $\partial M$ is supposed to be a compact, connected future (resp. past) converging $(n-1)$-manifold of non-positive mean curvature which satisfies in addition $\Theta<\frac{1}{2} \sqrt{C_{n} \mathscr{Y}}$. Notice that the CMC assumption simplifies the expression of the vector field $\mathbf{k}$ which becomes $\mathbf{k}=-\left(\operatorname{tr}_{\ell} \theta\right) e_{0}+k(v)$.

We will make some conformal change of metric and second fundamental form à la Choquet Bruhat-Lichnerowicz-York. The first step is to find a background conformal factor that we will denote by $u$ and that will be used in order to study the Choquet Bruhat-Lichnerowicz-York conformal change.

\subsection{A background conformal factor}

Consider the Lagrangian on $W_{-\frac{(n-2)}{2}}^{1,2}$ defined by

$$
\begin{aligned}
Q(f)= & \frac{1}{2} \int_{M}|\nabla f|^{2}+\frac{1}{2 C_{n}} \int_{M} \operatorname{Scal}^{g}(1+f)^{2}-\frac{1}{C_{n}} \int_{\partial M} \operatorname{tr}_{\ell} \theta(1+f)^{2} \\
& +\frac{1}{2} \sqrt{\frac{\mathscr{Y}}{C_{n}}\|1+f\|_{L^{\frac{C_{n}}{2}}(\partial M)}^{2},}
\end{aligned}
$$

which is non-negative, since $\operatorname{tr}_{\ell} \theta \leq 0$ on the boundary $\partial M$. In the same way as in [16], we can prove the

Proposition 3.1 There exists some $u \in W_{-\frac{(n-2)}{2}}^{1,2}$ such that $Q(u)=\min Q$. Moreover, the function $u$ satisfies $0<1+u \leq 1$.

Proof The non-negativity of $Q$ implies that

$$
0 \leq \inf _{\substack{1,2 \\ W_{-\frac{(n-2)}{2}}}} Q<+\infty
$$

Let us consider $\left(u_{i}\right)_{i \in \mathbb{N}}$ a minimizing sequence. It is clear that $Q$ is coercive in $W_{-\frac{(n-2)}{2}}^{1,2}$, so that $\left(u_{i}\right)_{i \in \mathbb{N}}$ is bounded in $W_{-\frac{(n-2)}{2}}^{1,2}$. Since $W_{-\frac{(n-2)}{2}}^{1,2} \hookrightarrow L^{2}(\partial M)$ and $W_{-\frac{(n-2)}{2}}^{1,2} \hookrightarrow$ $L_{\eta-\frac{(n-2)}{2}}^{2}(M)$ with $\eta>0$ are compact injections, there exists a sub-sequence (still denoted by $\left.\left(u_{i}\right)_{i}\right)$ that converges strongly in $L_{\eta-\frac{(n-2)}{2}}^{2}(M)$ and in $L^{2}(\partial M)$ towards 
some $u \in W_{-\frac{(n-2)}{2}}^{1,2}$ (remark that the convergence can be supposed pointwise almost everywhere as well). Using Fatou's lemma, we obtain

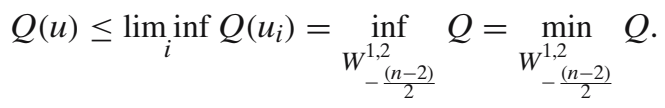

Following the arguments of $[16,17]$, we can prove by contradiction that the function $u$ cannot be identically -1 on the boundary. So assume it is the case, and consider the solution $h \in W_{\alpha}^{2, p}$ (with $\left.-\frac{(n-2)}{2}-1<\alpha \leq-\frac{(n-2)}{2}\right)$ of the boundary problem

$$
\begin{array}{r}
\Delta h+\frac{1}{C_{n}} h \mathrm{Scal}^{g}=0 \text { on } M, \\
\mathrm{~d} h(v)=-1 \text { on } \partial M,
\end{array}
$$

where $\Delta$ denotes the positive Laplace operator with respect to $g$. We know that $h$ is a positive function and has the following asymptotic expansion $h=\frac{\kappa}{r^{(n-2)}}+h_{1}$, where $\kappa>0$ and $h_{1}=o\left(\frac{1}{r^{(n-2)}}\right)$. Let us take $\varepsilon>0$ and compute

$$
\begin{aligned}
Q(u+\varepsilon h)-Q(u) & =\varepsilon\left\{\int_{M}(1+u)\left(\Delta_{g} h+\frac{1}{C_{n}} \mathrm{Scal}^{g} h\right)+\lim _{\infty} \int_{S_{r}(b)} \mathrm{d} h\left(v_{r}\right)\right\}+O\left(\varepsilon^{2}\right) \\
& =-\varepsilon(n-2) \kappa \operatorname{Vol}\left(\mathbb{S}^{n-1}, g_{\mathbb{S}^{n-1}}\right)+O\left(\varepsilon^{2}\right),
\end{aligned}
$$

where $\operatorname{Vol}\left(\mathbb{S}^{n-1}, g_{\mathbb{S}^{n-1}}\right)$ denotes the volume of the round sphere of radius 1 . Consequently for small positive values of $\varepsilon$, one gets $Q(u+\varepsilon h)-Q(u)<0$ which is in contradiction with the minimizing character of $u$ in $W_{-\frac{(n-2)}{2}}^{1,2}$.

As a matter of fact, since it is not identically -1 on the boundary, our minimizer $u \in W_{-\frac{(n-2)}{2}}^{1,2}$ satisfies the Euler-Lagrange equations associated to $Q$ that is to say

$$
\begin{array}{cc}
\Delta_{g} u+\frac{1}{C_{n}} \mathrm{Scal}^{g}(1+u)=0 & \text { on } M, \\
\mathrm{~d} u(v)=\sqrt{\frac{\mathscr{Y}}{C_{n}}}\|1+u\|_{L^{\frac{C_{n}}{2}}(\partial M)}^{-\frac{2}{n-2}}(1+u)^{\frac{n}{n-2}}-\frac{2}{C_{n}} \operatorname{tr}_{\ell} \theta(1+u) & \text { on } \partial M .
\end{array}
$$

Since the function $u$ is superharmonic in a neighborhood of its minimum, this one is consequently achieved on the boundary. Then suppose there exists some point $y \in \partial M$ such that $u(y) \leq-1$. Using the boundary equation satisfied by $u$ one gets, at the point $y$

$$
\mathrm{d} u(v)=\sqrt{\frac{\mathscr{Y}}{C_{n}}}\|1+u\|_{L^{\frac{C_{n}}{2}(\partial M)}}^{-\frac{2}{n-2}}(1+u)^{\frac{n}{n-2}}-\frac{2}{C_{n}} \operatorname{tr}_{\ell} \theta(1+u) \leq 0,
$$

which is in contradiction with the maximum principle.

We have to prove now that $u \leq 0$. Let us consider again $\varepsilon>0$ small enough and define $u_{\varepsilon}=(u-\varepsilon)^{+}=\max \{0, u-\varepsilon\} \geq 0$. Since $u$ tends to 0 at infinity, we know that $u_{\varepsilon}$ is compactly supported on $M$, and on $\operatorname{Supp} u_{\varepsilon}$ we clearly have $u_{\varepsilon}=u-\varepsilon$ and 
$\nabla u_{\varepsilon}=\nabla u$. Then we compute, using Stokes' theorem

$$
\begin{aligned}
\int_{M}\left|\nabla u_{\varepsilon}\right|^{2} & =\int_{M}\left\langle\nabla u_{\varepsilon}, \nabla u\right\rangle \\
& =\int_{M} u_{\varepsilon} \Delta_{g} u-\int_{\partial M} u_{\varepsilon} \mathrm{d} u(v) \\
& \leq 0,
\end{aligned}
$$

since $\Delta_{g} u \leq 0$ on $M$, and $\mathrm{d} u(v) \geq 0$ on $\partial M$. Since $M$ is connected, we obtain the vanishing of $u_{\varepsilon}$ which means $u \leq \varepsilon$ for every $\varepsilon>0$ small enough. We can conclude that $u \leq 0$ by sending $\varepsilon$ to 0 .

In the remainder of this text, we consider some positive number $p$ satisfying the inequality $\max \left\{2, \frac{n}{2}\right\}<p \leq n$ in order to obtain the continuous injection $W_{\tau}^{2, p} \subset C_{\tau}^{0, \alpha}$. Then $u$ is actually $W_{\tau^{\prime}}^{2, p}$ for some $\tau<\tau^{\prime}<-\frac{(n-2)}{2}$, because of the elliptic regularity of the Laplace operator, since the metric satisfies $(g-b) \in C_{\tau}^{2, \alpha} \subset W_{\tau}^{2, p}$. This is obtained with local inequalities, Bartnik's scaling argument (cf. Proposition 1.15 of [2]) and the use of relevant cutoff functions. Thereby $u$ is $C_{\tau^{\prime}}^{0, \alpha}$ and so $C_{\tau^{\prime \prime}}^{2, \alpha}$ because of the elliptic regularity of the Laplace operator in the Hölder classes, where $\tau^{\prime}<\tau^{\prime \prime}<-\frac{(n-2)}{2}$.

\subsection{A modified conformal change}

Let us consider the conformally modified couple of metric and second fundamental form $(\bar{g}, \bar{k})=\left(\varphi^{4 /(n-2)} g, \zeta \varphi^{-2} k\right)$ where $\varphi=1+v, v \in W_{\beta}^{2, p}, \beta<-\frac{(n-2)}{2}$. In this section, the constant $\zeta$ only satisfies the condition $\zeta>0$. Some transformation formulae are collected in the

Lemma 3.2 If $(\bar{g}, \bar{k})=\left(\varphi^{4 /(n-2)} g, \zeta \varphi^{-2} k\right)$ then

$$
\begin{aligned}
& \Phi(\bar{g}, \bar{k})=\left(\begin{array}{c}
\varphi^{-\frac{n+2}{n-2}}\left(C_{n} \Delta_{g} \varphi+\mathrm{Scal}^{g} \varphi\right)-\zeta^{2} \varphi^{-\frac{4 n}{n-2}}|k|_{g}^{2} \\
2 \zeta \varphi^{-\frac{2 n}{n-2}} \delta_{g} k
\end{array}\right) \\
& \operatorname{tr}_{\bar{\ell}} \bar{\theta}=\varphi^{-\frac{2}{n-2}} \operatorname{tr}_{\ell} \theta+\left(\frac{C_{n}}{2}\right) \varphi^{-\frac{n}{n-2}} \mathrm{~d} \varphi(v) \\
& |\bar{k}(\bar{v})|_{\bar{g}}=\zeta \varphi^{-\frac{2 n}{n-2}}|k(v)|_{g} \\
& \bar{m}=m-\frac{C_{n}}{16 \pi} \lim _{\infty} \int_{S_{r}(b)} \mathrm{d} v\left(v_{r}\right) \mathrm{dVol}_{S_{r}(b)} \\
& \overline{p^{i}}=\zeta p^{i} \text {. }
\end{aligned}
$$

Proof Cf. $[6,8,12,25]$ for the conformal transformation of the constraints. The transformation formula for $m$ has been proved in [16,17] and the one for $p^{i}$ is straightforward since $v \in W_{\beta}^{2, p}$, and $\beta<-\frac{(n-2)}{2}$.

We are interested in the following problem $(P)$ : finding some $v \in W_{-\frac{(n-2)}{2}}^{2, p}$ such that

$$
\begin{array}{ll}
\Delta_{g} v+\frac{1}{C_{n}} \mathrm{Scal}^{g}(1+v)-\frac{\zeta^{2}}{C_{n}}|k|_{g}^{2}(1+v)^{-\frac{3 n-2}{n-2}}=\frac{2 \zeta}{C_{n}}(1+v)^{-\frac{n}{n-2}}\left|\delta_{g} k\right|_{g} & \text { on } M, \\
\operatorname{tr}_{\bar{\ell}} \bar{\theta}+|\bar{k}(\bar{v})|_{\bar{g}}=\frac{1}{2} \operatorname{Vol}(\partial M, \bar{g})^{\frac{-1}{n-1}} \sqrt{C_{n} \mathscr{Y}(\partial M, \bar{\ell})} & \text { on } \partial M .
\end{array}
$$


This problem is quite natural since it consists on taking the equality cases of respectively the dominant energy condition (remark nonetheless that we do not solve the constraints equations) and the boundary condition of Theorem 1.1 with respect to the couple $(\bar{g}, \bar{k})$. According to the formulae of Lemma 3.2 and in virtue of the conformal invariance of the Yamabe number $\mathscr{Y},(P)$ is equivalent to

$$
\begin{aligned}
& \Delta_{g} v+\frac{1}{C_{n}} \operatorname{Scal}^{g}(1+v)-\frac{2 \zeta}{C_{n}}\left|\delta_{g} k\right|_{g}(1+v)^{-\frac{n}{n-2}}-\frac{\zeta^{2}}{C_{n}}|k|_{g}^{2}(1+v)^{-\frac{3 n-2}{n-2}}=0, \\
& \mathrm{~d} v(v)=\sqrt{\frac{\mathscr{Y}}{C_{n}}\|1+v\|_{L}^{-\frac{C_{n}}{2}}(\partial M)}(1+v)^{\frac{n}{n-2}}-\frac{2}{C_{n}} \operatorname{tr}_{\ell} \theta(1+v)-\frac{2 \zeta}{C_{n}}|k(v)|_{g}(1+v)^{-\frac{n}{n-2}} .
\end{aligned}
$$

The main result of this section is the following existence theorem.

Theorem 3.3 There exists a weak solution $v \in W_{-\frac{(n-2)}{2}}^{1,2}$ (not necessary unique) to the problem $(P)$, which satisfies $u \leq v \leq 0$ almost everywhere on $M$.

Proof We will prove the existence of a (pointwise) decreasing sequence $\left(u_{i}\right)_{i \in \mathbb{N}}$ of $W_{-\frac{(n-2)}{2}}^{2, p}$ defined by

$$
\left\{\begin{array}{c}
u_{0}=0 \\
\forall i \in \mathbb{N}^{*}, u_{i} \geq u \text { solution of }\left(P_{i}\right)
\end{array}\right.
$$

where $\left(P_{i}\right)$ is the following boundary problem

$$
\begin{aligned}
\Delta_{g} f+\mathcal{H}(f) & =0, \\
-\mathrm{d} f(v)+\mathcal{G}_{i}(f) & =0,
\end{aligned}
$$

where we have set

$$
\begin{aligned}
\mathcal{H}(f)= & \frac{1}{C_{n}} \operatorname{Scal}^{g}(1+f)-\frac{2 \zeta}{C_{n}}\left|\delta_{g} k\right|_{g}(1+f)^{-\frac{n}{n-2}}-\frac{\zeta^{2}}{C_{n}}|k|_{g}^{2}(1+f)^{-\frac{3 n-2}{n-2}}, \\
\mathcal{G}_{i}(f)= & \sqrt{\frac{\mathscr{Y}}{C_{n}}\left\|1+u_{i-1}\right\|_{L^{\frac{C_{n}}{2}}(\partial M)}^{-\frac{2}{n-2}}(1+f)^{\frac{n}{n-2}}-\frac{2}{C_{n}} \operatorname{tr}_{\ell} \theta(1+f),} \\
& -\frac{2 \zeta}{C_{n}}|k(v)|_{g}(1+f)^{-\frac{n}{n-2}} .
\end{aligned}
$$

If we define the following Lagrangians

$$
\begin{aligned}
E_{i}(f)= & \frac{1}{2} \int_{M}|\nabla f|^{2}+\frac{1}{2 C_{n}} \int_{M} \operatorname{Scal}^{g}(1+f)^{2}+\frac{(n-2) \zeta}{C_{n}} \int_{M}\left|\delta_{g} k\right|_{g}(1+f)^{-\frac{2}{n-2}} \\
& +\frac{(n-2) \zeta^{2}}{2 n C_{n}} \int_{M}|k|_{g}^{2}(1+f)^{-\frac{2 n}{n-2}}+\frac{(n-2) \zeta}{C_{n}} \int_{\partial M}|k(v)|_{g}(1+f)^{-\frac{2}{n-2}} \\
& -\frac{1}{C_{n}} \int_{\partial M} \operatorname{tr}_{\ell} \theta(1+f)^{2}+\left.2 \sqrt{\frac{\mathscr{Y}}{C_{n}^{3}}}\left\|1+u_{i-1}\right\|\right|_{L^{\frac{C_{n}}{2}}(\partial M)} ^{-\frac{2}{\partial}} \int_{\partial M}(1+f)^{\frac{C_{n}}{2}}
\end{aligned}
$$

on $\mathcal{F}_{i}:=\left\{f \in W_{-\frac{(n-2)}{2}}^{1,2} / u \leq f \leq u_{i-1}\right\}$. Then the equations of $\left(P_{i}\right)$ are the formal Euler-Lagrange equations of $E_{i}$. Since we have assumed that $\operatorname{tr}_{\ell} \theta \leq 0$ we know that each $E_{i}$ is non-negative and coercive with respect to the $W_{-\frac{(n-2)}{2}}^{1,2}$-norm (nonetheless, our assumptions do not allow us the conclusion that $E_{i}$ is finite or even differentiable 
on the whole $\left.W_{-\frac{(n-2)}{2}}^{1,2}\right)$. However, let us suppose that $u_{0}=0 \geq u_{1} \geq \cdots \geq u_{i-1} \geq u$ are given strong solutions of respectively $\left(P_{1}\right),\left(P_{2}\right), \ldots,\left(P_{i-1}\right)$. We are going to construct the $(i+1)$ th term of the sequence $\left(u_{i}\right)_{i}$. We verify we can apply Theorem 1.2 of [32]. The Hilbert space $W_{-\frac{(n-2)}{2}}^{1,2}$ is reflexive and its subspace $\mathcal{F}_{i}$ is closed (for the pointwise convergence) and convex (closed [for the pointwise convergence] and convex subsets of Banach spaces are a large class of weakly closed sets which are needed for Theorem 1.2 of [32]). Since $E_{i}$ is coercive, the only thing to verify is the weak lower semicontinuity of $E_{i}$ on $\mathcal{F}_{i}$. Let us take $\left(v_{m}\right)_{m \in \mathbb{N}} \subset \mathcal{F}_{i}$ a sequence which weakly converges in $W_{-\frac{(n-2)}{2}}^{1,2}$ to some $u_{i} \in \mathcal{F}_{i}$. Passing to a sub-sequence if necessary, we can assume that $v_{m} \stackrel{m}{\longrightarrow} u_{i}$ pointwise almost everywhere. Then using Fatou's lemma and also the lower semi-continuity of the $W_{-\frac{(n-2)}{2}}^{1,2}$-norm, we can conclude that

$$
E_{i}\left(u_{i}\right) \leq \liminf _{m} E_{i}\left(v_{m}\right) .
$$

In virtue of Theorem 1.2 of [32], $E_{i}$ is bounded on $\mathcal{F}_{i}$ and attains its infimum on $\mathcal{F}_{i}$. Let us denote by $u_{i}$ a minimizer. The harder is then to prove that $u_{i}$ weakly solves the problem $\left(P_{i}\right)$.

We first claim that $u$ and $u_{i-1}$ are respectively a sub-solution and a super-solution of $\left(P_{i}\right)$. Indeed we compute on one hand

$$
\begin{aligned}
\left(\Delta_{g}+\mathcal{H}\right)(u)= & -\frac{\zeta}{C_{n}}\left|\delta_{g} k\right|_{g}(1+u)^{-\frac{n}{n-2}}-\frac{\zeta^{2}}{C_{n}}|k|_{g}^{2}(1+u)^{-\frac{3 n-2}{n-2}} \\
\leq & 0 \\
-\mathrm{d} u(v)+\mathcal{G}_{i}(u)= & \sqrt{\frac{\mathscr{Y}}{C_{n}}}(1+u)^{\frac{n}{n-2}}\left(\left\|1+u_{i-1}\right\|_{L^{\frac{C_{n}}{2}(\partial M)}}^{-\frac{2}{n_{n}}}-\|1+u\|_{L^{\frac{C_{n}}{2}(\partial M)}}^{-\frac{2}{2}}\right) \\
& -\frac{2 \zeta}{C_{n}}|k(v)|_{g}(1+u)^{-\frac{n}{n-2}} \\
\leq & 0,
\end{aligned}
$$

since $1+u>0$ and $u \leq u_{i-1}$. On the other hand we obtain

$$
\begin{aligned}
\left(\Delta_{g}+\mathcal{H}\right)\left(u_{i-1}\right)= & 0 \\
-\mathrm{d} u_{i-1}(\nu)+\mathcal{G}_{i}\left(u_{i-1}\right)= & \sqrt{\frac{\mathscr{Y}}{C_{n}}\left(1+u_{i-1}\right)^{\frac{n}{n-2}}\left\|1+u_{i-1}\right\|_{L^{\frac{C_{n}}{2}}(\partial M)}^{-\frac{2}{n-2}}} \\
& -\sqrt{\frac{\mathscr{Y}}{C_{n}}\left(1+u_{i-1}\right)^{\frac{n}{n-2}}\left\|1+u_{i-2}\right\|_{L^{\frac{C_{n}}{2}}(\partial M)}^{-\frac{2}{n^{2}}}} \\
& +\underbrace{\left(-\mathrm{d} u_{i-1}(\nu)+\mathcal{G}_{i-1}\left(u_{i-1}\right)\right)}_{=0} \\
\geq & 0
\end{aligned}
$$

since $u_{i-1}$ is a strong solution of $P_{i-1},\left(1+u_{i-1}\right)>(1+u)>0$, and $u_{i-1} \leq u_{i-2}$ by assumption.

In the following, we denote by $\mathscr{D} E_{i}=\left(\mathscr{D}^{M} \oplus \mathscr{D}^{\partial M}\right) E_{i}$ the formal derivative of $E_{i}$ whose action on any test function $\varphi$ will be denoted by

$$
\left\langle\varphi, \mathscr{D} E_{i}(f)\right\rangle=\left\langle\varphi, \mathscr{D}^{M} E_{i}(f)\right\rangle+\left\langle\varphi, \mathscr{D}^{\partial M} E_{i}(f)\right\rangle .
$$


Now take some $\varphi \in C_{0}^{\infty}(M)$, some $\varepsilon>0$ small enough, and define the function $v_{\varepsilon}=u_{i}+\varepsilon \varphi-\varphi^{\varepsilon}+\varphi_{\varepsilon}$, where

$$
\varphi^{\varepsilon}=\max \left\{0, u_{i}+\varepsilon \varphi-u_{i-1}\right\} \geq 0 \text { and } \varphi_{\varepsilon}=-\min \left\{0, u_{i}+\varepsilon \varphi-u\right\} \geq 0
$$

are compactly supported (actually $\left[\operatorname{Supp}\left(\varphi^{\varepsilon}\right) \cup \operatorname{Supp}\left(\varphi_{\varepsilon}\right)\right] \subset \operatorname{Supp}(\varphi)$ ) functions since $u \leq u_{i} \leq u_{i-1}$ by construction. We know that $u_{i}$ minimizes $E_{i}$ in $\mathcal{F}_{i}$ so that we have

$$
0 \leq\left\langle\left(v_{\varepsilon}-u_{i}\right), \mathscr{D} E_{i}\left(u_{i}\right)\right\rangle=\varepsilon\left\langle\varphi, \mathscr{D} E_{i}\left(u_{i}\right)\right\rangle+\left\langle\varphi_{\varepsilon}, \mathscr{D} E_{i}\left(u_{i}\right)\right\rangle-\left\langle\varphi^{\varepsilon}, \mathscr{D} E_{i}\left(u_{i}\right)\right\rangle,
$$

so that

$$
\left\langle\varphi, \mathscr{D} E_{i}\left(u_{i}\right)\right\rangle \geq \frac{1}{\varepsilon}\left(\left\langle\varphi^{\varepsilon}, \mathscr{D} E_{i}\left(u_{i}\right)\right\rangle-\left\langle\varphi_{\varepsilon}, \mathscr{D} E_{i}\left(u_{i}\right)\right\rangle\right)
$$

Since $u_{i-1}$ is a super-solution of $\left(P_{i}\right)$ and $\varphi^{\varepsilon} \geq 0$, we obtain on one hand

$$
\begin{aligned}
\left\langle\varphi^{\varepsilon}, \mathscr{D} E_{i}\left(u_{i}\right)\right\rangle \geq & \left\langle\varphi^{\varepsilon}, \mathscr{D} E_{i}\left(u_{i}\right)\right\rangle-\int_{M} \varphi^{\varepsilon}\left(\Delta_{g}+\mathcal{H}\right)\left(u_{i-1}\right) \\
& -\int_{\partial M} \varphi^{\varepsilon}\left(-\mathrm{d} u_{i-1}(v)+\mathcal{G}_{i}\left(u_{i-1}\right)\right) \\
= & \int_{M}\left\langle\nabla \varphi^{\varepsilon}, \nabla\left(u_{i}-u_{i-1}\right)\right\rangle+\int_{M} \varphi^{\varepsilon}\left(\mathcal{H}\left(u_{i}\right)-\mathcal{H}\left(u_{i-1}\right)\right) \\
& +\int_{\partial M} \varphi^{\varepsilon}\left(\mathcal{G}_{i}\left(u_{i}\right)-\mathcal{G}_{i}\left(u_{i-1}\right)\right) \\
= & \int_{M \cap \operatorname{Supp}\left(\varphi^{\varepsilon}\right)}\left|\nabla\left(u_{i}-u_{i-1}\right)\right|^{2} \\
& +\int_{M \cap \operatorname{Supp}\left(\varphi^{\varepsilon}\right)}\left(u_{i}-u_{i-1}\right)\left(\mathcal{H}\left(u_{i}\right)-\mathcal{H}\left(u_{i-1}\right)\right) \\
& +\int_{\partial M \cap \operatorname{Supp}\left(\varphi^{\varepsilon}\right)}\left(u_{i}-u_{i-1}\right)\left(\mathcal{G}_{i}\left(u_{i}\right)-\mathcal{G}_{i}\left(u_{i-1}\right)\right) \\
& +\varepsilon \int_{M \cap \operatorname{Supp}\left(\varphi^{\varepsilon}\right)}\left\langle\nabla \varphi, \nabla\left(u_{i}-u_{i-1}\right)\right\rangle \\
& +\varepsilon \int_{M \cap \operatorname{Supp}\left(\varphi^{\varepsilon}\right)} \varphi\left(\mathcal{H}\left(u_{i}\right)-\mathcal{H}\left(u_{i-1}\right)\right) \\
& +\varepsilon \int_{\partial M \cap \operatorname{Supp}\left(\varphi^{\varepsilon}\right)} \varphi\left(\mathcal{G}_{i}\left(u_{i}\right)-\mathcal{G}_{i}\left(u_{i-1}\right)\right) \\
\geq & o(\varepsilon) .
\end{aligned}
$$

The first equality is an application of Stokes' theorem (which is authorized since $u_{i-1}$ is a $W_{-\frac{(n-2)}{2}}^{2,2}$ solution of $\left.P_{i-1}\right)$, whereas the second one is given by the explicit expression of $\varphi^{\varepsilon}$ on its support. The last inequality results from the increasing character of the functionals $\mathcal{H}$ and $\mathcal{G}_{i}$, and on the fact that $\left|\operatorname{Supp}\left(\varphi^{\varepsilon}\right)\right|_{M}$ tends to 0 as $\varepsilon \longrightarrow 0$. 
On the other hand, since $u$ is a sub-solution of $\left(P_{i}\right)$ and $\varphi_{\varepsilon} \geq 0$, we get

$$
\begin{aligned}
-\left\langle\varphi_{\varepsilon}, \mathscr{D} E_{i}\left(u_{i}\right)\right\rangle \geq & -\left\langle\varphi_{\varepsilon}, \mathscr{D} E_{i}\left(u_{i}\right)\right\rangle+\int_{M} \varphi^{\varepsilon}\left(\Delta_{g}+\mathcal{H}\right)(u) \\
& +\int_{\partial M} \varphi^{\varepsilon}\left(-\mathrm{d} u(v)+\mathcal{G}_{i}(u)\right) \\
= & \int_{M}\left\langle\nabla \varphi_{\varepsilon}, \nabla\left(u-u_{i}\right)\right\rangle+\int_{M} \varphi_{\varepsilon}\left(\mathcal{H}(u)-\mathcal{H}\left(u_{i}\right)\right) \\
& +\int_{\partial M} \varphi_{\varepsilon}\left(\mathcal{G}_{i}(u)-\mathcal{G}_{i}\left(u_{i}\right)\right) \\
= & \int_{M \cap \operatorname{Supp}\left(\varphi_{\varepsilon}\right)}\left|\nabla\left(u-u_{i}\right)\right|^{2} \\
& +\int_{M \cap \operatorname{Supp}\left(\varphi_{\varepsilon}\right)}\left(u-u_{i}\right)\left(\mathcal{H}(u)-\mathcal{H}\left(u_{i}\right)\right) \\
& +\int_{\partial M \cap \operatorname{Supp}\left(\varphi_{\varepsilon}\right)}\left(u-u_{i}\right)\left(\mathcal{G}_{i}(u)-\mathcal{G}_{i}\left(u_{i}\right)\right) \\
& -\varepsilon \int_{M \cap \operatorname{Supp}\left(\varphi_{\varepsilon}\right)}\left\langle\nabla \varphi, \nabla\left(u-u_{i}\right)\right\rangle \\
& -\varepsilon \int_{M \cap \operatorname{Supp}\left(\varphi_{\varepsilon}\right)} \varphi\left(\mathcal{H}(u)-\mathcal{H}\left(u_{i}\right)\right) \\
& -\varepsilon \int_{\partial M \cap \operatorname{Supp}\left(\varphi_{\varepsilon}\right)} \varphi\left(\mathcal{G}_{i}(u)-\mathcal{G}_{i}\left(u_{i}\right)\right) \\
\geq & o(\varepsilon) .
\end{aligned}
$$

The first equality is still an application of Stokes' theorem (which is authorized as well, since our background conformal factor $u$ is a $C_{\tau}^{2, \alpha}$ function for some $\left.\tau<-\frac{(n-2)}{2}\right)$, whereas the second one is given by the explicit expression of $\varphi_{\varepsilon}$ on its support. The last inequality results once again from the increasing character of the functionals $\mathcal{H}$ and $\mathcal{G}_{i}$, and on the fact that $\left|\operatorname{Supp}\left(\varphi_{\varepsilon}\right)\right|_{M}$ tends to 0 as $\varepsilon \longrightarrow 0$.

Thereby it follows that $\left\langle\varphi, \mathscr{D} E_{i}\left(u_{i}\right)\right\rangle \geq \frac{o(\varepsilon)}{\varepsilon} \longrightarrow 0$ as $\varepsilon \longrightarrow 0$. Reversing the sign of the test function $\varphi$ we obtain

$$
\left\langle\varphi, \mathscr{D} E_{i}\left(u_{i}\right)\right\rangle=0
$$

which exactly means that $u_{i}$ weakly solves $\left(P_{i}\right)$. Using one more time the elliptic regularity of the Laplace operator, it can be proved that the function $u_{i}$ is actually $W_{\tau^{\prime}}^{2, p}$ since $(g, k)$ satisfy $(g-b) \in C_{\tau}^{2, \alpha} \subset W_{\tau}^{2, p}, k \in C_{\tau-1}^{1, \alpha} \subset W_{\tau-1}^{1, p}$, where $\tau<\tau^{\prime}<-\frac{(n-2)}{2}$. It then comes out that $u_{i}$ is a strong solution of $\left(P_{i}\right)$, and so the existence of the sequence $\left(u_{i}\right)_{i \in \mathbb{N}}$ is finally achieved.

We will now show that the sequence $\left(u_{i}\right)_{i \in \mathbb{N}}$ is bounded in $W_{-\frac{(n-2)}{2}}^{1,2}$. Since $u_{i}$ minimizes the functional $E_{i}$ along the subset $\mathcal{F}_{i} \ni u$ we have 


$$
\begin{aligned}
E_{i}\left(u_{i}\right) \leq & E_{i}(u) \\
\leq & \frac{1}{2} \int_{M}|\nabla u|^{2}+\frac{1}{2 C_{n}} \int_{M} \mathrm{Scal}^{g}(1+u)^{2}+\frac{(n-2) \zeta}{C_{n}} \int_{M}\left|\delta_{g} k\right|_{g}(1+u)^{-\frac{2}{n-2}} \\
& +\frac{(n-2) \zeta^{2}}{2 n C_{n}} \int_{M}|k|_{g}^{2}(1+u)^{-\frac{2 n}{n-2}}+\frac{(n-2) \zeta}{C_{n}} \int_{\partial M}|k(v)|_{g}(1+u)^{-\frac{2}{n-2}} \\
& -\frac{1}{C_{n}} \int_{\partial M} \operatorname{tr}_{\ell} \theta(1+u)^{2}+2 \sqrt{\frac{\mathscr{Y}}{C_{n}^{3}}}\|1+u\|_{L^{\frac{C_{n}}{2}}(\partial M)}^{2} \\
= & K(u)<\infty,
\end{aligned}
$$

where $K(u)$ is finite (it is a consequence of $(1+u) \in C^{2}, 0<1+u \leq 1$ on $M$, and the $L^{1}$ character of $\left.\mathrm{Scal}^{g},\left|\delta_{g} k\right|_{g},|k|_{g}^{2}\right)$ and does not depend upon $i$. This last inequality gives the boundedness of $\left(u_{i}\right)_{i}$ by coercivity of $E_{i}$ with respect to the $W_{-\frac{(n-2)}{2}}^{1,2}$-norm. Passing to a sub-sequence if necessary we can assume that $\left(u_{i}\right)_{i}$ weakly converges in $W_{-\frac{(n-2)}{2}}^{1,2}$ towards some $v$. We will finally prove that $v$ is a weak solution of $(P)$. We have for any test function $\varphi$

$$
\begin{aligned}
\int_{M}\langle\nabla v, \nabla \varphi\rangle= & \lim _{i \longrightarrow \infty} \int_{M}\left\langle\nabla u_{i}, \nabla \varphi\right\rangle \\
= & \lim _{i \rightarrow \infty} \int_{M} \varphi \Delta_{g} u_{i}-\int_{\partial M} \varphi \mathrm{d} u_{i}(v) \\
= & -\lim _{i \longrightarrow \infty}\left\{\int_{M} \varphi \mathcal{H}\left(u_{i}\right)+\int_{\partial M} \varphi \mathcal{G}_{i}\left(u_{i}\right)\right\} \\
= & -\int_{M} \varphi \mathcal{H}(v)-\sqrt{\frac{\mathscr{Y}}{C_{n}}}\|1+v\| \|^{-\frac{2}{n-2}} \int_{\partial M} \varphi(1+v)^{\frac{n}{n-2}} \\
& +\frac{2}{C_{n}} \int_{\partial M} \varphi \operatorname{tr}_{\ell} \theta(1+v)-\frac{2 \zeta}{C_{n}} \int_{\partial M} \varphi|k(v)|_{g}(1+v)^{-\frac{n}{n-2}} .
\end{aligned}
$$

The first equality is obvious by the definition of the weak convergence of $\left(u_{i}\right)_{i}$ towards $v$, and the second and third ones are clear since $u_{i}$ is a $W^{2, p}$ solution of $P_{i}$. The last one is a consequence of Lebesgue's theorem on dominated convergence. We then conclude that $v$ is a weak solution of $(P)$.

Remark 3.4 Reasoning in the same way as for $u$ with the elliptic regularity of the Laplace operator, we can notice that $v$ is actually $W_{\tau^{\prime}}^{2, p}$ and so $C_{\tau^{\prime \prime}}^{2, \alpha}$, for some $\tau<$ $\tau^{\prime}<\tau^{\prime \prime}<-\frac{(n-2)}{2}$, since $(g, k)$ satisfy $(g-b) \in C_{\tau}^{2, \alpha} \subset W_{\tau}^{2, p}, k \in C_{\tau-1}^{1, \alpha} \subset W_{\tau-1}^{1, p}$. As a consequence the inequality $-1<u \leq v \leq 0$ holds everywhere on $M$.

\section{End of the proofs}

We firstly give the proof of Theorem 1.2.

Theorem 1.2 Let $(M, g, k)$ be a $n$-dimensional, complete, $C_{\tau}^{2, \alpha}$-asymptotically flat $\left(\tau<-\frac{n-2}{2}, n \geq 3\right), C M C$ and spin manifold with constraints satisfying the dominant energy condition and $\Phi(g, k) \in L^{1}\left(M, \mathrm{dVol}_{g}\right)$. Suppose furthermore that $M$ has a

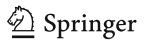


compact, connected and weakly future (resp. past) trapped boundary $\partial M$ of positive Yamabe invariant and of non-positive mean curvature satisfying

$$
\Theta<\frac{1}{2} \sqrt{C_{n} \mathscr{Y}}
$$

Then there exists (an explicit definition will be given below) a small positive constant $\zeta_{0}=\zeta_{0}(M, g)$, such that $p^{\mu}=\left(m, p^{i}\right)$ the energy-momentum of $(g, k)$ satisfies

$$
m \geq \zeta_{0} \sqrt{\sum_{i=1}^{n}\left(p^{i}\right)^{2}}+\frac{\mathfrak{S}\left(\sqrt{\mathscr{Y} C_{n}}-2 \Theta\right)}{\mathfrak{S}+\left(\sqrt{\mathscr{Y} C_{n}}-2 \Theta\right)}\left(\frac{V^{\frac{4}{C_{n}}}}{16 \pi}\right)
$$

Proof We have constructed, in Sect. 3, thanks to a standard conformal method $(M, \bar{g}, \bar{k})=\left(M,(1+v)^{\frac{4}{n-2}} g, \zeta(1+v)^{-2} k\right)$ which is a $C_{\tau}^{2, \alpha}$-asymptotically flat manifold $\left(\tau<-\frac{(n-2)}{2}\right)$ satisfying the equality case of the dominant energy condition and the boundary equality

$$
\operatorname{tr}_{\bar{\ell}} \bar{\theta}+|\bar{k}(\bar{v})|_{\bar{g}}=\frac{1}{2} \operatorname{Vol}(\partial M, \bar{\ell})^{\frac{-1}{n-1}} \sqrt{C_{n} \mathscr{Y}(\partial M, \bar{\ell})} .
$$

Hence $(M, \bar{g}, \bar{k})$ satisfies all the assumptions of Theorem 1.1. In this section, we will give some restriction on the admissible positive parameter $\zeta$ by giving the definition of the geometric positive constant $\zeta_{0}(M, g)$.

Now, according to the conformal transformation formulae of Lemma 3.2 for the energy-momentum and applying Theorem 1.1, one gets

$$
\bar{m}=m-\frac{C_{n}}{16 \pi} \lim _{r \rightarrow \infty} \int_{S_{r}(b)} \mathrm{d} v\left(v_{r}\right) \mathrm{dVol}_{S_{r}(b)} \geq \sqrt{\sum_{i=1}^{n}\left(\overline{p^{i}}\right)^{2}}=\zeta \sqrt{\sum_{i=1}^{n}\left(p^{i}\right)^{2}} .
$$

But we remark that $\lim _{r \rightarrow \infty} \int_{S_{r}(b)} \mathrm{d} v\left(v_{r}\right)=\frac{1}{2} \lim _{r \rightarrow \infty} \int_{S_{r}(b)} \mathrm{d}\left((1+v)^{2}\right)\left(v_{r}\right)$ since $v=o\left(r^{-\frac{n-2}{2}}\right)$. Then applying Stokes' theorem, it follows

$$
\begin{aligned}
\lim _{r \rightarrow \infty} \int_{S_{r}(b)} \mathrm{d}\left((1+v)^{2}\right)\left(v_{r}\right)= & \int_{\partial M} \mathrm{~d}\left((1+v)^{2}\right)(v)+2 \int_{M}|\nabla v|^{2}+\frac{2}{C_{n}} \int_{M} \mathrm{Scal}^{g}(1+v)^{2} \\
& -\frac{4 \zeta}{C_{n}} \int_{M}\left|\delta_{g} k\right|_{g}(1+v)^{-\frac{2}{n-2}}-\frac{2 \zeta^{2}}{C_{n}} \int_{M}|k|_{g}^{2}(1+v)^{-\frac{2 n}{n-2}},
\end{aligned}
$$

but we know that

$$
\begin{aligned}
2(1+v) \mathrm{d}(1+v)(v)= & 2 \sqrt{\frac{\mathscr{Y}}{C_{n}}}\|1+v\|_{L^{\frac{C_{n}}{2}}(\partial M)}^{-\frac{2}{(\partial M}}(1+v)^{\frac{C_{n}}{2}} \\
& -\frac{4}{C_{n}} \operatorname{tr}_{\ell} \theta(1+v)^{2}-\frac{4 \zeta}{C_{n}}|k(v)|_{g}(1+v)^{-\frac{2}{n-2}},
\end{aligned}
$$


which entails

$$
\begin{aligned}
\lim _{r \rightarrow \infty} \int_{S_{r}(b)} \mathrm{d}\left((1+v)^{2}\right)\left(v_{r}\right)= & 2 \int_{M}|\nabla v|^{2}+\frac{2}{C_{n}} \int_{M} \mathrm{Scal}^{g}(1+v)^{2} \\
& -\frac{4 \zeta}{C_{n}} \int_{M}\left|\delta_{g} k\right|_{g}(1+v)^{-\frac{2}{n-2}}-\frac{2 \zeta^{2}}{C_{n}} \int_{M}|k|_{g}^{2}(1+v)^{-\frac{2 n}{n-2}} \\
& -\frac{4}{C_{n}} \int_{\partial M} \operatorname{tr}_{\ell} \theta(1+v)^{2}-\frac{4 \zeta}{C_{n}} \int_{\partial M}|k(v)|_{g}(1+v)^{-\frac{2}{n-2}} \\
& +2 \sqrt{\frac{\mathscr{Y}}{C_{n}}}\|1+v\|_{L^{\frac{C_{n}}{2}}(\partial M)} .
\end{aligned}
$$

It will be convenient to denote by $F(v)$ the right-hand side member of the equality above, in other words

$$
\begin{aligned}
F(v)= & 2 \int_{M}|\nabla v|^{2}+2 \sqrt{\frac{\mathscr{Y}}{C_{n}}}\|1+v\|^{2}{ }_{L}^{\frac{C_{n}}{2}}(\partial M) \\
& +\frac{2}{C_{n}} \int_{M}(1+v)^{2}\left(\mathrm{Scal}^{g}-2 \zeta\left|\delta_{g} k\right|_{g}(1+v)^{-\frac{C_{n}}{2}}-\zeta^{2}|k|_{g}^{2}(1+v)^{-C_{n}}\right) \\
& -\frac{4}{C_{n}} \int_{\partial M}(1+v)^{2}\left(\operatorname{tr}_{\ell} \theta+\zeta|k(v)|_{g}(1+v)^{-\frac{C_{n}}{2}}\right) .
\end{aligned}
$$

But now we can define the positive and scale-invariant constant $\zeta_{0}=\zeta_{0}(M, g)$ as

$$
\zeta_{0}(M, g):=\inf _{M}\left\{(1+u)^{\frac{C_{n}}{2}}\right\}>0
$$

since we proved in Proposition 3.1 that the continuous function $u \in C_{\tau^{\prime \prime}}^{2, \alpha}$ cannot achieve the value -1 neither on $M$ nor on the boundary $\partial M$. In that case, for every $\left.\zeta \in] 0, \zeta_{0}\right]$, we have

$$
\begin{aligned}
\mathrm{Scal}^{g}-\zeta\left|\delta_{g} k\right|_{g}(1+v)^{-\frac{C_{n}}{2}}-\zeta^{2}|k|_{g}^{2}(1+v)^{-C_{n}} & \geq 0 \\
\operatorname{tr}_{\ell} \theta+\zeta|k(v)|_{g}(1+v)^{-\frac{C_{n}}{2}} & \leq \operatorname{tr}_{\ell} \theta+|k(v)|_{g}
\end{aligned}
$$

in virtue of the dominant energy condition $\left(\mathrm{Scal}^{g}-2\left|\delta_{g} k\right|_{g}-|k|_{g}^{2} \geq 0\right.$ under the CMC assumption) and $u \leq v$. The assumption $\operatorname{tr}_{\ell} \theta+|k(v)|_{g} \leq \frac{1}{2} \operatorname{Vol}(\partial M, \ell)^{\frac{-1}{n-1}} \sqrt{C_{n} \mathscr{Y}}$ obviously implies

$$
\begin{aligned}
F(v) & \geq 2 \int_{M}|\nabla v|^{2}+2 \sqrt{\frac{\mathscr{Y}}{C_{n}}}\|1+v\|_{L^{\frac{C_{n}}{2}}(\partial M)}^{2}-\frac{4}{C_{n}} \int_{\partial M}(1+v)^{2}\left(\operatorname{tr}_{\ell} \theta+|k(v)|_{g}\right) \\
& \geq 2 \int_{M}|\nabla v|^{2}+\left(\frac{2}{C_{n}}\right) V^{\frac{-1}{n-1}}\left(\sqrt{\mathscr{Y} C_{n}}-2 \Theta\right) \int_{\partial M}(1+v)^{2}
\end{aligned}
$$

where we have used Hölder inequality. It follows using Young inequality: 


$$
\begin{aligned}
& \forall \varepsilon>0 \quad(1+v)^{2} \geq\left(1-\frac{1}{\varepsilon}\right)+(1-\varepsilon) v^{2}, \text { that } \\
& \qquad(v) \geq 2 \int_{M}|\nabla v|^{2}+\left(\frac{2}{C_{n}}\right) V^{\frac{-1}{n-1}}\left(\sqrt{\mathscr{Y} C_{n}}-2 \Theta\right)\left\{\left(1-\frac{1}{\varepsilon}\right) V+(1-\varepsilon) \int_{\partial M} v^{2}\right\} \\
&=2 \int_{M}|\nabla v|^{2}+\left(\frac{2}{C_{n}}\right)\left(\sqrt{\mathscr{Y} C_{n}}-2 \Theta\right)\left(1-\frac{1}{\varepsilon}\right) V^{\frac{4}{C_{n}}} \\
&+\left(\frac{2}{C_{n}}\right) V^{\frac{-1}{n-1}}\left(\sqrt{\mathscr{Y} C_{n}}-2 \Theta\right)(1-\varepsilon) \int_{\partial M} v^{2} .
\end{aligned}
$$

Suppose now that $F(v)<\eta\left(\frac{2}{C_{n}}\right)\left(\sqrt{\mathscr{Y} C_{n}}-2 \Theta\right) V^{\frac{4}{C_{n}}}$ where $\eta$ is a positive constant. Then we obtain

$$
\begin{aligned}
2 \int_{M}|\nabla v|^{2} & +\left(\frac{2}{C_{n}}\right) V^{\frac{-1}{n-1}}\left(\sqrt{\mathscr{Y} C_{n}}-2 \Theta\right)(1-\varepsilon) \int_{\partial M} v^{2} \\
& <\left(\frac{2}{C_{n}}\right)\left(\sqrt{\mathscr{Y} C_{n}}-2 \Theta\right)\left(\eta-1+\frac{1}{\varepsilon}\right) V^{\frac{4}{C_{n}}} .
\end{aligned}
$$

The left-hand side member of this inequality is non-negative if and only if

$$
\mathfrak{S}:=C_{n} V^{\frac{1}{n-1}} \inf _{W_{-\frac{(n-2)}{2}}^{1,2}}\left\{\frac{\int_{M}|\nabla f|^{2}}{\int_{\partial M} f^{2}}\right\} \geq\left(\sqrt{\mathscr{Y} C_{n}}-2 \Theta\right)(\varepsilon-1) .
$$

This condition entails $\eta>1-\frac{1}{\varepsilon}$. But this function of $\varepsilon$ achieves its maximum for $\varepsilon_{\max }=1+\frac{\mathfrak{S}}{\left(\sqrt{\mathscr{Y C} C_{n}}-2 \Theta\right)}$ and therefore $\eta>1-\frac{1}{\varepsilon_{\max }}$. By contraposition, if we take

$$
\eta=1-\frac{1}{\varepsilon_{\max }}=\frac{\mathfrak{S}}{\mathfrak{S}+\left(\sqrt{\mathscr{Y} C_{n}}-2 \Theta\right)},
$$

then $F(v) \geq \eta \sqrt{\frac{\mathscr{Y}}{C_{n}}} V^{\frac{4}{C_{n}}}$ and so it comes out

$$
m-\zeta \sqrt{\sum_{i=1}^{n}\left(p^{i}\right)^{2}} \geq \frac{C_{n}}{32 \pi} F(v) \geq \frac{\mathfrak{S}\left(\sqrt{\mathscr{Y} C_{n}}-2 \Theta\right)}{\mathfrak{S}+\left(\sqrt{\mathscr{Y} C_{n}}-2 \Theta\right)}\left(\frac{V^{\frac{4}{C_{n}}}}{16 \pi}\right),
$$

which gives finally the desired inequality.

We end this section with the proof of Theorem 1.4.

Theorem 1.4 Suppose that the following conditions are satisfied:

1. The assumptions of Theorem 1.2 hold,

2. $g$ and $k$ are smooth,

3. Additional technical assumption: the symmetric 2-tensor $k$ restricted to TวM vanishes, namely $k^{\partial M}=0$.

If equality is achieved in (1), then $k=0$ on the whole manifold $M$, and $(M, g)$ is isometric to the standard spacelike n-slice in the exterior Schwarzschild metric of mass $m, M=\mathbb{R}^{n} \backslash B(0, R)$ with

$$
R=\left(\frac{4 \pi m}{(n-1) \omega_{n-1}}\right)^{\frac{1}{n-2}} \text { and } g=\left(1+\left(\frac{R}{r}\right)^{n-2}\right)^{\frac{4}{n-2}} \text { eucl. }
$$


Proof When equality is achieved in (1), we obtain the null character of $\left(\bar{p}^{\mu}\right)$ the energy-momentum of $(\bar{g}, \bar{k})$. This means that each non-negative term of the integrated Bochner formula vanishes, for a certain (not for every) spinor field $\psi \in \operatorname{ker} \mathfrak{D}^{\gamma}$ which is asymptotic to some non-zero and constant at infinity spinor $\psi_{0}$

$$
\begin{aligned}
0 & =4 \pi\left\langle\psi_{0}, e_{0} \cdot \bar{p} \cdot \psi_{0}\right\rangle \\
& =\int_{M}\left(\left|\nabla^{\bar{\gamma}} \psi\right|^{2}+\langle\bar{\Re} \psi, \psi\rangle\right)+\int_{\partial M}\left\langle-\mathfrak{D}^{\bar{\ell}} \psi+\frac{1}{2} e_{0} \cdot \overline{\mathbf{k}} \cdot \psi, \psi\right\rangle .
\end{aligned}
$$

In particular, the boundary integral has no contribution when one computes $4 \pi\left\langle\psi_{0}, e_{0} \cdot \bar{p} \cdot \psi_{0}\right\rangle$. Since the manifold $(M, \bar{g}, \bar{k})$ is asymptotically flat, there exists a compact subset $K$ which contains the boundary $\partial M$, and a chart at infinity $\Upsilon$ : $M \backslash K \longrightarrow \mathscr{S}_{R}:=\mathbb{R}^{n} \backslash B(0, R)$ such that the energy-momentum of $\left(\mathscr{S}_{R}, \Upsilon_{*} \bar{g}, \Upsilon_{*} \bar{k}\right)$ is null. But the smoothness of $(g, k)$ implies (by bootstraping the regularity arguments used to prove that $v$ is $\left.C_{\tau}^{2, \alpha}\right)$ the smoothness of $\left(\Upsilon_{*} \bar{g}, \Upsilon_{*} \bar{k}\right)$ on $\mathscr{S}_{R}$. We can then apply Theorem 3.2 of [13] which asserts that $\left(\bar{p}^{\mu}\right)$ vanishes.

This fact has many consequences on the whole manifold $M$, since (4) is now true for a basis of spinor fields. Then there exists a basis $\mathscr{B} \subset\left(\Sigma \cap \operatorname{ker} P_{+}\right)$of $\nabla \bar{\gamma}-$ parallel spinor fields on $M$ which satisfy in addition $\left\langle\left(-\bar{\lambda}+\frac{1}{2} e_{0} \cdot \overline{\mathbf{k}}\right) \cdot \psi, \psi\right\rangle=0$, for any $\psi \in \mathscr{B}$. This entails the vanishing of the vector field $\left(-2 \bar{\lambda}-\operatorname{tr}_{\bar{\ell}} \bar{\theta}\right) e_{0}+\bar{k}(\bar{v})$ on the boundary, that is to say

$$
\operatorname{tr}_{\bar{\ell}} \bar{\theta}=2 \bar{\lambda} \text {, and } \bar{k}(\bar{v})=0 \text {. }
$$

On the other hand our conformal couple $(\bar{g}, \bar{k})$ satisfies along $\partial M$

$$
\operatorname{tr}_{\bar{\ell}} \bar{\theta}+|\bar{k}(\bar{\nu})|_{\bar{g}}=\frac{1}{2} \bar{V}^{\frac{-1}{n-1}} \sqrt{C_{n} \mathscr{Y}} \leq 2 \bar{\lambda},
$$

which means that we are in the equality case of the Bär-Hijazi estimate, and consequently $\bar{\ell}$ is Einstein with positive scalar curvature Scal ${ }^{\bar{\ell}}=\kappa^{2}(n-1)(n-2)$. Now the traced Gauss equation on $\partial M$ can be written as follows

$$
\operatorname{Ric}^{\bar{g}, \partial M}=\frac{\operatorname{Scal}^{\bar{\ell}}}{(n-1)} \bar{\ell}-\left(\operatorname{tr}_{\bar{\ell}} \bar{\theta}\right) \bar{\theta}+\bar{\theta} \circ \bar{\theta} .
$$

On the other hand, since $M$ has a basis of $\nabla \bar{\gamma}$-parallel spinor fields, one can show (see the spinorial curvature computations of $[13,23]$ ) that the curvature tensor $R^{\bar{\gamma}}$ vanishes and we get the following geometric equations

$$
\begin{aligned}
R^{\bar{g}} & =\frac{1}{2} \bar{k} \oslash \bar{k} \\
\mathrm{~d}^{\nabla^{\bar{g}}} \bar{k} & =0 .
\end{aligned}
$$

We get by taking a trace, that $\operatorname{Ric}^{\bar{g}}=-\left(\operatorname{tr}_{\bar{g}} \bar{k}\right) \bar{k}+\bar{k} \circ \bar{k}=\bar{k} \circ \bar{k}$ on the whole $M$. In particular, this equation leads, by restriction to $\partial M$,

$$
\operatorname{Ric}^{\bar{g}, \partial M}=\bar{k}^{\partial M} \circ \bar{k}^{\partial M}=\zeta_{0}^{2}(1+v)^{-4}\left(k^{\partial M} \circ k^{\partial M}\right)=0,
$$

thanks to the additional assumptions 3. Since $\bar{\ell}$ is a Yamabe metric on $\partial M$, the value of the mean cuvature is known to be $\operatorname{tr}_{\bar{\ell}} \bar{\theta}=\kappa(n-1)$, we then get

$$
\kappa^{2}(n-2) \bar{\ell}=\kappa(n-1) \bar{\theta}-\bar{\theta} \circ \bar{\theta} .
$$


We can remark that every eigenvalue $\mu_{i}$ of $\bar{\theta} \in S^{2}(T \partial M)$ is a root of the polynomial

$$
P(X)=X^{2}-\kappa(n-1) X+\kappa^{2}(n-2) .
$$

$P$ has two roots $\kappa$ and $(n-2) \kappa$. In the 3-dimensional case $n=3$, it is clear that the boundary $\partial M$ is totally umbillic in $\bar{g}(\bar{\theta}$ is proportional to $\bar{\ell})$. In higher dimensions, we notice once again that the mean curvature is $\operatorname{tr}_{\bar{\ell}} \bar{\theta}=\kappa(n-1)$ which imposes that every eigenvalue $\mu_{i}=\kappa$, and so $\partial M$ is totally umbillic in $\bar{g}$ as well. It comes out, by the Gauss equation

$$
R^{\bar{\ell}}=\frac{1}{2} \kappa^{2}(\bar{\ell} \oslash \bar{\ell})
$$

that the boundary $\partial M$ has constant sectional curvature and so is the quotient of the round sphere $\mathbb{S}^{n} / \Gamma$. As in [17], the Gromov-Heintze-Karcher inequality leads to the conclusion that the group $\Gamma$ is trivial. Now, we glue $M$ along the boundary $\partial M=\mathbb{S}^{n}$ with $\mathbb{B}=\left(B^{n}\left(0, \frac{1}{\kappa^{2}}\right), g_{\text {eucl }}, k=0\right)$, the $n$-dimensional flat ball of radius $\frac{1}{\kappa^{2}}$ endowed with a zero extrinsic curvature 2 -tensor. This produces $(\tilde{M}, \tilde{g}, \tilde{k})$ a complete, CMC, $n$-dimensional asymptotically flat spin manifold with zero energy-momentum. Hence, applying [13] again, $(\tilde{M}, \tilde{g}, \tilde{k})$ can be isometrically embeddable in Minkowski spacetime $\mathbb{R}^{n, 1}$. But the maximal hypersurfaces in $\mathbb{R}^{n, 1}$ have automatically vanishing second fundamental form (see the estimate of Theorem 3 of [11]), that is to say $\tilde{k}=0$ and so $k=0$. This means that we are in the equality case of [17], so that we can conclude that $(M, g)$ is a spacelike $n$-slice of the Schwarzschild metric.

Remark 4.3 The technical assumption 3 could appear rather artificial and restrictive, but it is actually natural in order to make the gluing construction with the manifold $\mathbb{B}$ which is the simplest that one could imagine. Indeed it seems quite hard to find (actually too hard for the author), for arbitrary traceless 2-tensors $k$, examples of topological balls endowed with a metric and extrinsic curvature tensor which glue well with $(M, \bar{g}, \bar{k})$.

Acknowledgements The author is grateful to M. Herzlich for having pointed out a mistake in an earlier version of this article, and also to P. T. Chruściel for many helpful discussions.

\section{Appendix}

The aim of this appendix is to show that the class of asymptotically flat spin manifolds under consideration in this paper is non-empty, and even quite large. The idea is to construct examples of couples $(g, k)$ satisfying the assumptions of Theorem 1.2 , by deforming the Schwarzschild metric that we will denote by $g_{S}$. We recall that $g_{S}$ is a scalar-flat and asymptotically flat metric which lies in the conformal class of the flat space, and has a minimal sphere as boundary. Let us take some function $\chi \in C_{\tau}^{0, \alpha}$, $\chi>0$ for some $\tau<-((n-2) / 2)$. The first step of our construction is to prove the existence of a regular solution to the problem

$$
\begin{aligned}
\Delta_{g_{S}} \varphi & =\chi, \\
\mathrm{d} \varphi\left(v_{g_{S}}\right) & =0,
\end{aligned}
$$

where $\Delta_{g_{S}}$ and $v_{g_{S}}$ are respectively the Laplace operator and boundary unit normal, both with respect to the Schwarzschild metric. This problem can be easily solved by 
applying Lax-Milgram theorem in the Hilbert space $W_{-\frac{(n-2)}{2}}^{1,2}$ to the scalar product $a$ defined by

$$
a\left(f_{1}, f_{2}\right):=\int_{M}\left\langle\mathrm{~d} f_{1}, \mathrm{~d} f_{2}\right\rangle,
$$

and to the continuous linear form $f \longmapsto \int_{M} \chi f$. Thanks to the elliptic regularity of the Laplace operator in the Hölder classes, $\varphi \in C_{\tau^{\prime}}^{2, \alpha}$ for some $\tau<\tau^{\prime}<-\frac{(n-2)}{2}$. We shall prove that our solution $\varphi$ is actually positive on $M$. To this end, set $\varphi_{\varepsilon}=\min \{0, \varphi+\varepsilon\}$, for any $\varepsilon>0$. Obviously, $\varphi_{\varepsilon}$ is a compactly supported ( since $\varphi \stackrel{\infty}{\longrightarrow} 0$ ) non-positive function, and a simple computation leads to

$$
\begin{aligned}
\int_{M}\left|\nabla \varphi_{\varepsilon}\right|^{2} & =\int_{M}\left\langle\nabla \varphi_{\varepsilon}, \nabla \varphi\right\rangle \\
& =\int_{M} \varphi_{\varepsilon} \chi \\
& \leq 0
\end{aligned}
$$

Therefore $\nabla \varphi_{\varepsilon} \equiv 0$ which merely implies $\varphi_{\varepsilon} \equiv 0$, since $M$ is assumed to be connected. It follows that $\varphi+\varepsilon \geq 0$ for every $\varepsilon>0$, and we can conclude that $\varphi \geq 0$ by sending $\varepsilon$ to 0 . We finally remark that $\varphi$ cannot vanish because of the strong version of the maximum principle.

Now we define the metric $g:=(1+\varphi)^{\frac{4}{n-2}} g_{S}$ which, in virtue of the conformal transformation formulae of Lemma 3.2 and of the equalities above, satisfies

$$
\begin{aligned}
\mathrm{Scal}^{g} & =C_{n}(1+\varphi)^{-\frac{(n+2)}{(n-2)}} \chi \\
\operatorname{tr}_{\ell} \theta & =0 .
\end{aligned}
$$

We have thus constructed, by conformal deformation from the Schwarzschild metric, another metric which has positive scalar curvature and a minimal spherical boundary.

The second step is to find a suitable extrinsic curvature tensor. We will make it by solving the elliptic TT-tensor problem (TT) on vector field [25]

$$
\begin{array}{r}
\Delta_{g} X=\delta_{g} \delta_{0}^{*} X=0 \text { on } M \\
\left(\delta_{0}^{*} X\right)(v)=\omega \nu \text { on } \partial M,
\end{array}
$$

where $\omega \in C^{0}(\partial M)$. Then $(T T)$ has a unique solution $X$ and we get a relevant extrinsic curvature tensor by defining $k:=\delta_{0}^{*} X$ which is de facto traceless and divergence free.

The remaining question is: how can we obtain the necessary conditions

$$
\Theta=V^{\frac{1}{n-1}} \sup _{\partial M}|\omega| \leq \frac{1}{2} \sqrt{C_{n} \mathscr{Y}\left(\mathbb{S}^{n-1}, g_{\mathbb{S}^{n-1}}\right)} \quad \text { and } \quad \mathrm{Scal}^{g} \geq|k|_{g}^{2} ?
$$

The ellipticity of (TT) implies a control $\left\||k|_{g}^{2}\right\|_{C_{\tau}^{2, \alpha}} \leq C\|\omega\|_{C^{0}(\partial M)}$, and choosing $\|\omega\|_{C^{0}(\partial M)}$ small enough will allow us to obtain (5) since $g$ has positive scalar curvature, and the Yamabe number of the standard sphere is positive. Thus we have singled out a large class of couples $(g, k)$ satisfying all the assumptions of Theorem 1.2. 


\section{References}

1. Bär, C.: Lower eigenvalues estimates for Dirac operators. Math. Ann. 293, 39-46 (1992)

2. Bartnik, R.: The mass of an asymptotically flat manifold. Comm. Pure Appl. Math. 39, 661-693 (1986)

3. Bartnik, R.: Existence of maximal surfaces in asymptotically flat spacetimes. Commun. Math. Phys. 94, 155-175 (1984)

4. Bartnik, R., Chruściel, P.T.: Boundary value problems for Dirac type equations. J. Reine Angew. Math. 579, 13-73 (2005)

5. Bartnik, R., Chruściel, P.T., O’Murchadha, N.: On maximal surfaces in asymptotically flat spacetimes. Commun. Math. Phys. 130, 95-109 (1990)

6. Bartnik, R., Isenberg, J.: The Constraint Equations, The Einstein Equations and the Large Scale Behavior of Gravitational Fields, 50 years of the Cauchy Problem in General Relativity, Birkhäuser.

7. Beig, R., Chruściel, P.T.: Killing vectors in asymptotically flat space-times: I. Asymptotically translationnal Killing vectors and the rigid positive energy theorem. J. Math. Phys. 37, 1939-1961 (1996)

8. Besse, A.L.: Einstein manifolds. Springer (1987)

9. Bray, H.: Proof of the Riemannian Penrose inequality using the positive mass theorem. J. Differential Geom. 59(2), 117-267 (2001)

10. Bray, H., Chruściel, P.T.: The Penrose inequality, The Einstein Equations and the Large Scale Behavior of Gravitational Fields, 50 years of the Cauchy Problem in General Relativity, Birkhäuser (2004)

11. Cheng, S.-Y., Yau, S.-T.: Maximal space-like hypersurfaces in the Lorentz-Minkowski spaces. Ann. Math. 104, 407-419 (1976)

12. Choquet-Bruhat, Y., Isenberg, J., York, J.W.: Einstein constraints on asymptotically Euclidean manifolds. Phys. Rev. D(3), 61(8) (2000)

13. Chruściel, P.T., Maerten, D.: Killing vectors in asymptotically flat space-times: II. Asymptotically translationnal Killing vectors and the rigid positive energy theorem in higher dimensions, to appear in J. Math. Phys.

14. Galloway, G.J., Schoen, R.: A generalization of Hawking's black hole topology theorem to higher dimensions, gr-qc/0509107.

15. Gibbons, G.W., Hawking, S.W., Horrowitz, G.T., Perry, M.J.: Positive mass theorem for black holes. Commun. Math. Phys. 88, 295-308 (1983)

16. Herzlich, M.: A Penrose-like inequality for the mass of Riemannian asymptotically flat manifolds. Commun. Math. Phys. 188, 121-133

17. Herzlich, M.: Minimal Surfaces, the Dirac Operator and the Penrose Inequality. Séminaire de Théorie Spectrale et Géométrie GRENOBLE 20, 9-16 (2002)

18. Herzlich, M.: The positive mass theorem for black holes revisited. J. Geom. Phys. 26, 97-111 (1998)

19. Hijazi, O.: Première valeur propre de l'opérateur de Dirac et nombre de Yamabe. C. R. Acad. Sci. Paris 313, 865-868 (1991)

20. Huisken, G., Ilmanen, T.: The Riemannian Penrose inequality. Internat. Math. Res. Notices 20, 1045-1058 (1997)

21. Huisken, G., Ilmanen, T.: The inverse mean curvature flow and the Riemannian Penrose inequality. J. Diffrential Geom. 59(3), 353-437 (2001)

22. Lawson, H.B., Michelson, M.L.: Spin Geometry. Princeton (1989)

23. Maerten, D.: The positive energy-momentum theorem in AdS-asymptotically hyperbolic manifolds. Ann. Henri Poincaré 7(5), 975-1011 (2006)

24. Malec, E., Mars, M., Simon, W.: On the Penrose Inequality for general horizons, grqc/02011024 v2 (2002)

25. Maxwell, D.: Solutions of the Einstein constraints equations with apparent horizon boundaries, gr-qc/0307117 v3

26. O’Neill, B.: Semi-Riemannian geometry. Academic Press, San Diego (1983)

27. Parker, T., Taubes, C.: On Witten's proof of the positive energy theorem. Commun. Math. Phys. 84, 223-238 (1982)

28. Penrose, R.: Naked singularities. Ann. N. Y. Acad. Sci. 224, 125-134 (1973)

29. Schoen, R., Yau, S.-T.: On the proof of the positive mass conjecture in general relativity. Commun. Math. Phys. 65, 45-76 (1979)

30. Schoen, R., Yau, S.-T.: The energy and the linear momentum of space-times in general relativity. Commun. Math. Phys. 79, 47-51 (1981) 
31. Schoen, R., Yau, S.-T.: Proof of the positive mass theorem II. Commun. Math. Phys. 79, 231-260 (1981)

32. Struwe, M.: Variational Methods, vol. 34. Springer (2000)

33. Wald, R.: General relativity. University press of Chicago (1984)

34. Weinstein, G., Yamada, S.: On a Penrose inequality with charge. preprint, math.DG/0405602

35. Witten, E.: A simple proof of the positive energy theorem. Commun. Math. Phys. 80, 381-402 (1981) 